\title{
Safinamide: a new hope for Parkinson's disease?
}

\section{Fábio G. Teixeira ${ }^{1,2,}$, Miguel F. Gago ${ }^{1,2,3, \ddagger}$, Paulo Marques ${ }^{1,2}$, Pedro Silva Moreira ${ }^{1,2}$, Ricardo Magalhães ${ }^{1,2}$, Nuno Sousa ${ }^{1,2}$ and António J. Salgado, ${ }^{1,2}$}

\footnotetext{
${ }^{1}$ Life and Health Sciences Research Institute (ICVS), School of Medicine, University of Minho, Braga, Portugal

${ }^{2}$ ICVS/3Bs - PT Government Associate Laboratory, Braga/Guimarães, Portugal

${ }^{3}$ Neurology Department, Hospital da Senhora da Oliveira, EPE, Guimarães, Portugal
}

The loss of dopaminergic neurons (DAn) and reduced dopamine (DA) production underlies the reasoning behind the gold standard treatment for Parkinson's disease (PD) using levodopa (L-DOPA). Recently licensed by the European Medicine Agency (EMA) and US Food and Drug Administration (FDA), safinamide [a monoamine oxidase B (MOA-B) inhibitor] is an alternative to L-DOPA; as we discuss here, it enhances dopaminergic transmission with decreased secondary effects compared with L-DOPA. In addition, nondopaminergic actions (neuroprotective effects) have been reported, with safinamide inhibiting glutamate release and sodium/calcium channels, reducing the excitotoxic input to dopaminergic neuronal death. Effects of safinamide have been correlated with the amelioration of nonmotor symptoms (NMS), although these remain under discussion. Overall, safinamide can be considered to have potential antidyskinetic and neuroprotective effects and future trials and/or studies should be performed to provide further evidence for its potential as an anti-PD drug.

\section{Introduction}

PD is the second most-common chronic neurodegenerative disorder worldwide, characterized by the degeneration of DAn and deficient DA production in the nigrostriatal pathway [1,2]. Clinically, it is recognized by a core of motor symptoms, including bradykinesia, rigidity, tremor, and postural instability, which are used to establish its diagnosis [3]. Although it has been postulated that PD itself is not a primary cause of death, studies have proposed that death might occur as a secondary result of the progression of PD motor dysfunctions, for example, as a result of falls in patients with advanced-stage PD [4-6]. In addition, the development of NMS, such as sleep disturbances, depression, olfactory dysfunction, and behavioral and/or cognitive problems, has also been linked with functional disabilities [7-10]. Therefore, management

Corresponding author: Teixeira, F.G. (fabioteixeira@med.uminho.pt)

$\ddagger$ These authors contributed equally to this work. strategies have been preferred, involving the diagnosis and evaluation of the condition of the patient, followed by the development and application of personalized strategies, aiming to ameliorate the patient's quality of life [11-13]. Still, as recently reviewed by Onofrj and colleagues [14], satisfactory approaches to relieve the symptoms, or slow down the progression, of PD by protecting DAn from premature death remain lacking. Promising results have been experimentally and clinically obtained with several drugs, yet the challenge remains to show a clinical proof of arrest of delay of DAn loss in PD.

The current symptomatic treatment relies on the use of pharmacological strategies, such as L-DOPA, which remains the gold standard treatment, DA agonists (DAAs; e.g., ropinirole or pramipexole), and MAO-B (e.g., rasagiline or selegiline) and catechol-Omethyltransferase (COMT; e.g., entacapone or tolcapone) inhibitors, to compensate for the deficits of DA in the nigrostriatal dopaminergic pathway [15-17] (Fig. 1). Although efficacious, they 


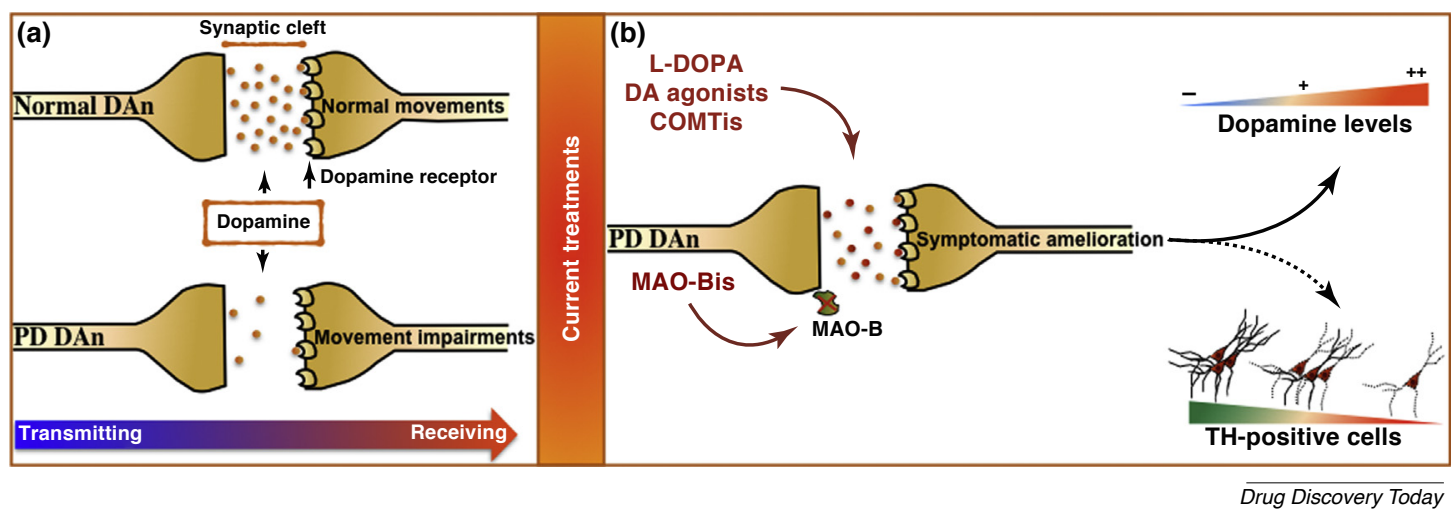

FIGURE 1

Current Parkinson's disease (PD) treatments. Considering PD as a single diagnostic entity because of the (a) loss of dopamine (DA) was and has been effective in the development and application of (the available) (b) symptomatic treatments (e.g., DA level re-establishment), but insufficient to stop PD progression through neuroprotective or disease-modifying actions. Abbreviations: COMTis, catechol-O-methyltransferase inhibitors; DAn, dopaminergic neuron; L-DOPA, levodopa; MAO-Bis, monoamine oxidase B inhibitors; $\mathrm{TH}$, tyrosine hydroxylase.

have several adverse effects (AEs), which can limit their use over long periods of time [14]. In addition, although surgical interventions, such as deep brain stimulation (DBS), have also been used as strategies for the treatment of $\mathrm{PD}$, the apparent clinical recovery is not effective in the long-term and the progression of the disease is not halted [18]. Molecular agents (e.g., mGluR4 agonists; CEP1347; GM1 ganglioside, CoQ10, and NAC) and gene engineering approaches [e.g., induction of glutamic acid decarboxylase (GAD) enzyme in the striatum; delivery of synthetic enzymes to increase striatal DA levels, and local infusion of neurotrophic factors to protect and restore nigral DA neurons] have also been developed and used in the treatment of PD [19-26]. However, none of the currently available strategies delay or halt its progression. The main reason for the failure of these approaches could be partly explained by the simplistic view and/or targeting and inadequate definition of PD [27]. In fact, in addition to DAn degeneration and DA depletion, multiple factors, including oxidative stress, mitochondrial dysfunction, excitotoxicity, and inflammation, have also been described as being involved in the initiation and progression of PD. This makes PD a multitargeted disease in which drugs, with a multimodal action, could be of particular value [28]. Thus, safinamide was recently proposed as a promising new multimodal PD drug [29]. Here, we discuss the current understanding of safinamide, addressing its therapeutical potential in preclinical and/or clinical applications, ongoing clinical trials and future prospects.

\section{Safinamide}

(S)-2-[[4-[(3-fluorophenyl)methoxy]phenyl]methyl]aminopropanamide methanesulfonate, safinamide, is a new molecule approved by the European Economic Area (EEA), EMA, and FDA as 'an add-on therapy to stable dose L-DOPA, alone or in combination with other PD therapies in mid-to late-stage-fluctuating PD patients' [30]. Safinamide has been described as a multimodal drug, combining dopaminergic and nondopaminergic actions, although it remains unclear how the nondopaminergic properties contribute to its global effect [31,32]. Whereas the dopaminergic action of safinamide leads to increased DA levels in the brain through its highly selective and reversible inhibition of MAO-B, its nondopaminergic actions have been correlated with the blocking of voltage-gated sodium channels and modulation of N-type calcium channels, with the consequent inhibition of excessive glutamate release [30,33]. In addition, although safinamide has been correlated with a linear pharmacokinetic after single or repeated administrations (suggesting that it is involved in enzyme inhibition or induction), the capacities of the amidases involved in its metabolism have not yet been fully characterized [30].

Safinamide at doses of 50-200 mg/day are safe and well tolerated, with low incidences of AEs compared with placebo [30]. Moreover, it shares a comparable efficacy with entacapone in reducing motor fluctuations, but with greater tolerability, including reduced nausea, vomiting, shortness of breath, urine abnormalities, and dizziness [34]. However, the concomitant consumption of safinamide has been associated with: (i) increased risk of fractures and falls when used with anxiolytics and antihypertensive drugs; (ii) increased risk of psychoses with amantadine use; and (iii) increased risk of neuropsychiatric AEs in patients taking DAAs [30]. The potential interactions of safinamide with amantadine and selective serotonin reuptake inhibitors (SSRIs) could also pose some limitations in clinical practice, especially in late stages of PD, given that serious AEs have been reported for other MAO-B inhibitors when used in combination with SSRIs and other antidepressants. These doubts could be mitigated by growing clinical practice and during postmarketing surveillance.

Thus, although safinamide differs from the currently MAO-B inhibitors rasagiline and selegiline because of its reversible mode of MAO-B inhibition, the clinical relevance of this difference is still not fully understood [30,32,35]. The reversible mode of safinamide represents an advantage, because studies have suggested that unspecific inhibition of MAO leads to unmetabolized dietary amines entering the circulatory system, where they induce noradrenaline release from peripheral adrenergic neurons, leading (in most cases) to a severe and potentially fatal hypertensive response. Therefore, to avoid such effects, MAO-B inhibitors with high selectivity, such as safinamide, are crucial for the 
treatment of PD $[14,36]$. In fact, studies have suggested that safinamide exhibits potent, highly selective, and reversible MAO-B inhibition (with full inhibition in human platelets at $0.6 \mathrm{mg} / \mathrm{kg}$ orally) [35,37], with its selectivity for MAO-B being relatively superior to that of selegiline and rasagiline, thus decreasing the need for dietary restrictions, such as the 'cheese effect' $[30,37]$. Binding studies in rat brain mitochondria (in vitro) demonstrated that safinamide has 5000 times greater selectivity for MAO-B (and 1000-fold selectivity over MAO-A in rat and human brains, respectively $[30,35,38]$ ) compared with 127 times and 103 times greater selectivity for MAO-B over MAO-A with selegiline and rasagiline, respectively $[30,36,39]$. Interestingly, in addition to such greater selectivity, MAO-B inhibition by safinamide is also fully reversible, because it does not form irreversible covalent bonds with MAO-B, in comparison with selegiline and rasagiline $[31,40,41]$. This is an important advantage for patients who experience AEs that need to be treated with other drugs, because the reversibility of safinamide avoids potential drug interactions [42-45]. From the (dosage) administration point of view, safinamide is water soluble and rapidly absorbed; by using a single oral dose of $100 \mathrm{mg}$ safinamide, a maximum plasma concentration was found after approximately $2 \mathrm{~h}$, being approximately $650 \mathrm{ng} / \mathrm{ml}$ and the area under the curve (AUC) being $19000 \mathrm{ng} / \mathrm{ml} \mathrm{h} \mathrm{[30].} \mathrm{In} \mathrm{addition,} \mathrm{although} \mathrm{several} \mathrm{studies} \mathrm{have}$ documented a secondary peak approximately 45 min after the first peak, safinamide has an elimination half-life of 21-24 h, allowing once-daily administration, with food intake not found to affect its absorption or bioavailability [33]. Indeed, as demonstrated by studies in animal models, brain levels of safinamide were higher than the corresponding plasma concentrations, demonstrating that the drug is largely biotransformed, also supported by the very low dosage of unchanged safinamide found in urine and feces; its plasma protein binding is $92 \%$, and only a small proportion is excreted unchanged [33]. This could be relevant for patients with $\mathrm{PD}$, because its effect quickly disappears, unlike selegiline or rasagiline, whose actions take several weeks to washout $[30,31,46]$. In addition, although studies with animal models have provided neuroprotective and neurorescuing properties of safinamide (which might result from its nondopaminergic actions), no evidence from human studies have confirmed these neuroprotective effects $[29,37]$. Thus, definitive conclusions on the efficacy and tolerability of safinamide compared with other PD drugs (e.g., selegiline, rasagiline, entacapone, or amantadine) are not yet possible, highlighting the need for additional research to fully elucidate the potential (neuroprotective) effect of safinamide in patients with PD [29].

\section{Preclinical evidence for the efficacy of safinamide in PD}

Espay and colleagues [27] assumed that approaching PD as a single diagnostic entity (e.g., dopamine-dependent disease) would be effective in the development of symptomatic therapies, but notably ineffective when targeting neuroprotection or attempting disease-modifying strategies. Such inefficacy might be explained in part by the simplistic single-target approach that has been established for drug development [27]. Nevertheless, the MAO-B inhibitors selegiline and rasagiline have been described as being able to mitigate PD progression $[47,48]$. Still, their neuroprotective action potential has been surrounded by controversy [49-52]. While for selegiline, studies have yet to reach a definitive conclusion $[53,54]$, the use of rasagiline in preclinical and/or clinical studies demonstrated a delayed and reduced need for the future use of L-DOPA [55-57]. However, there are still difficulties in documenting disease-modifying and/or neuroprotective effects when considering a PD drug with a symptomatic benefit [57]. Therefore, safinamide has been suggested to demonstrate efficacy as a multitargeted PD drug $[32,58]$.

Preclinical models of PD have demonstrated that, in addition to the symptomatic benefits of safinamide in PD resulting from the increase in DA levels through MAO-B inhibition, its nondopaminergic actions, including the attenuation of sodium/calcium channels and glutamate release, could lead to neuroprotective effects [37] (Fig. 2). In addition, neurorescuing and tremorolytic characteristics were also observed in PD animal models [59], as well as a reduction in the duration and intensity of L-DOPA-induced dyskinesia in parkinsonian nonhuman primates [60]. More recently, anti-inflammatory properties of safinamide have also been evidenced, with a role in blocking sodium channels in the activation of microglia, thereby suggesting that safinamide could reduce microglial activation and inflammation [28,61]. It has also been shown that safinamide enhances the development of a phagocytic phenotype in microglia in vitro, by improving the expression of arginase-1, a feature of a neuroprotective microglial phenotype known as ' $\mathrm{M} 2$ ' [28]. Notably, the changes in microglial phenotypes depend on the disease stage and severity. Therefore, as recently reviewed by Tang and Le [62], mastering the stage-specific switching of M1/M2 phenotypes within appropriate time windows could provide better therapeutic benefit for PD. Thus, the suppression of microglial activation together with the other nondopaminergic activities of safinamide (Fig. 2) could be clinically relevant, although more studies should be performed to address such hypotheses.

\section{Clinical evidence for the efficacy of safinamide in PD}

Most treatment strategies for PD act by increasing dopaminergic activity. L-DOPA remains the most-effective therapeutic approach and the gold standard treatment in PD [63]. Other therapies, such as COMT and MAO-B inhibitors, are used as add-on therapies to L-DOPA to increase the level of its unmetabolized form [63]. Until later stages of the disease, this strategy is effective in improving the motor symptoms and, therefore, treating the wearing-off phenomena, until later stages of the disease. However, long-term treatment with L-DOPA leads to troublesome motor fluctuations and/or L-DOPA-induced motor complications (MC; e.g., dyskinesias) [64]. In addition, the presence of NMS also causes significant clinical impairments, with the added difficulty of presenting a nonlinear clinical progression [2]. Thus, both dopaminergic and nondopaminergic strategies have to be tackled and combined during the course of the disease, a scenario in which safinamide is a promising choice [37]. Currently, 37 trials have been conducted on safinamide use, comprising 20 Phase I trials, nine Phase II trials, and eight Phase III trials [30]. Here, we provide a critical summary of some of these studies, paying particular attention to their inclusion criteria, design, and outcomes, and addressing outstanding questions that call for further studies. 


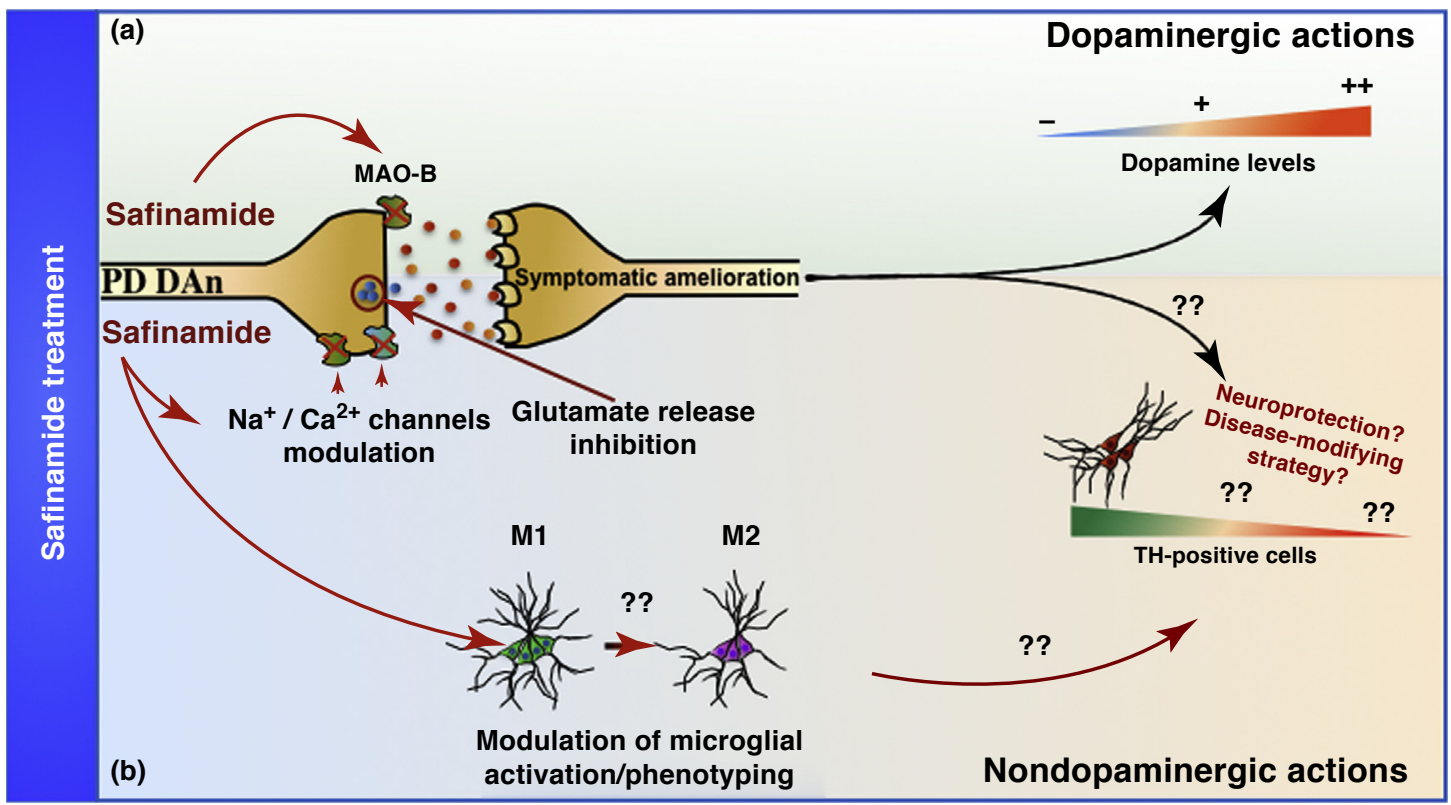

Drug Discovery Today

FIGURE 2

The multimodal profile of safinamide. (a) The reversible inhibition profile of monoamine oxidase B (MAO-B) by safinamide either alone or as an add-on to levodopa (L-DOPA) has been correlated with improvements in Parkinson's disease (PD) symptoms, increasing dopaminergic neuronal transmission because of the consequent increase in dopamine availability. In contrast to the majority of available PD drugs, (b) neuroprotective and disease-modifying effects of safinamide have also been claimed because of its capacity to block voltage-dependent sodium and N-type calcium channels, therefore inhibiting glutamate release. In addition, the modulation and/or suppression of microglial activation has also been linked to safinamide effects, suggesting an anti-inflammatory profile, which could be clinically important, given that microglia are implicated dopaminergic neuronal loss. Abbreviation: $\mathrm{TH}$, tyrosine hydroxylase.

\section{De novo, early-stage, drug-naïve PD}

To the best of our knowledge, there is only one study in 'de novo' (drug-naïve) 'early-stage' PD (Hoehn and Yahr Scale (HY) stage I or II; Study 009) [65]. In this Phase II study, safinamide (median dose of $70 \mathrm{mg} /$ day; range: $40-90 \mathrm{mg} /$ day) provided a reduction of at least $30 \%$ in the UPDRS-III score (only significant in the higher dose group) compared with $21.4 \%$ of placebo patients. However, this study had a follow-up period of only 3 months ( 12 weeks), and the dose of safinamide was, on average, low and adjusted to the body weight ( 0.5 or $1 \mathrm{mg} / \mathrm{kg}$ ), instead of a fixed 50/100/200 mg dosage.

\section{Intermediate stages of $P D$, without motor complications}

In intermediate stages of PD (patients with HY stage I-III with a disease duration of less than 5 years and already treated by DAAs but without L-DOPA-induced MCs), three Phase III studies have been published: Study 015 [66], Study 017 [67], and MOTION study [68]. These studies only screened patients with PD taking DAAs and, as such, patients taking L-DOPA have not yet been investigated.

In the Study 015, which was a 12-month double-blind study, patients with PD received a stable dose of a single DAA, and were then randomized to one daily dose of 100 or $200 \mathrm{mg}$ of safinamide, or a placebo. The group receiving $100 \mathrm{mg}$ of safinamide showed the most-significant improvements in their UPDRS III score ( -3.90 for $200 \mathrm{mg},-6.0$ for $100 \mathrm{mg}$, and -3.60 for placebo), Clinical Global Impression Change from baseline (CGI-C; +0.98 for $200 \mathrm{mg},+3.1$ for $100 \mathrm{mg}$, and 3.4 for the placebo group), and activities of daily life (UPDRS-II; -1.4 for $200 \mathrm{mg},-2.2$ for $100 \mathrm{mg}$, and 1.2 for the placebo group). However, a higher dose of $200 \mathrm{mg}$ of safinamide did not demonstrate any significant improvement.

Study 017 [67], a 12-month, randomized, double-blinded, placebo-controlled study of 227 patients with $\mathrm{PD}$, was an extension of Study 015. The primary efficacy endpoint was the time from baseline (Study 015 randomization) to 'intervention', defined as: increase in DAA dose; addition of another DAA, L-DOPA, or other PD treatment; or discontinuation because of a lack of efficacy. From the pooled data, and in terms of the safinamide-treated groups, the primary endpoint failed to reach statistical significance. Yet, post-hoc analyses revealed that patients receiving $100 \mathrm{mg}$ /day of safinamide experienced a significantly lower rate of intervention (25\% versus $51 \%$, respectively) and a delay in median time to intervention of 9 days (240-540-day analysis) compared with placebo.

Finally, the MOTION study [68], a Phase III, 24-week, doubleblind placebo-controlled trial of 679 patients with PD, explored the efficacy and safety of doses of 50 and $100 \mathrm{mg}$ /day of safinamide as an add-on therapy to a single stable DAA. The results showed that $100 \mathrm{mg}$ /day significantly improved PD motor symptoms (UPDRS III) and activities of daily living.

\section{Advanced-stage of PD with motor complications}

In patients with advanced-stage PD and L-DOPA-induced MC, three randomized, double-blind, placebo-controlled studies have examined the efficacy of safinamide: Study 016 [69], Study 018 
[70], and the SETTLE study [71] and, more recently, the clinical trial NCT00627640 [72].

In Study 016, 669 patients with PD (with an off-duration greater than $1.5 \mathrm{~h}$ /day) were randomized to either doses of 50 or $100 \mathrm{mg} /$ day of safinamide, or placebo for 3 months [69]. The addition of safinamide to L-DOPA significantly increased total on-time with no or nontroublesome dyskinesia $(1.36 \pm 2.63 \mathrm{~h}$ and $1.37 \pm 2.75 \mathrm{~h}$, respectively, compared with $0.97 \pm 2.38 \mathrm{~h}$ observed in placebo group). In addition, a decreased off time and improved motor symptoms (UPDRS-III) and Clinical Global ImpressionChange (CGI-C) were also observed [69].

In Study 018, 554 patients with PD who had completed Study 016 were enrolled and randomized to doses of 50 or $100 \mathrm{mg} /$ day of safinamide or to a placebo over a period of 6 and 18 months, with a total of 2 years of follow-up [70]. The primary endpoint of this study was set as the change in Dyskinesia Rating Scale (DRS) total score during on-time over 6 months. Although the safinamide groups presented a decrease in the mean of the total DRS score, significant differences were not observed compared with the placebo group. Study 018 further supported findings already evident from Study 016, showing significant clinical benefits (e.g., on-time without troublesome dyskinesia, off-time, activities of daily living, motor symptoms, quality of life, and symptoms of depression), which were found to be improved at 6 months and remained significant even after 2 years of follow-up.

In the SETTLE study [71], 549 patients with advanced PD were randomized for placebo or 50-100 $\mathrm{mg}$ safinamide as an add-on therapy to a stable dose of L-DOPA, for 6 months. As in Study 016, improvements were observed in the mean on-time with no or nontroublesome dyskinesia. Notably, a greater proportion of patients treated with safinamide $50-100 \mathrm{mg}$ had an improvement in both on- and off-time (either when considering the $\geq 30$ min or $\geq 60$-min cut off in both on- and off-time and a $\geq 20 \%$ or $\geq 30 \%$ improvement in the UPDRS III scores, respectively). Interestingly, the same clinical benefits were shown in Study 016 and Study 018, reinforcing the effects of safinamide on improving motor symptoms, CGI-C, Patient's Global Impression of Change, activities of daily living and health-related quality of life (Parkinson's Disease Questionnaire-39 and EuroQol-5 Dimensions). However, as in Study 018, the severity of dyskinesias (DRS score) did not improve. In addition, most of the patients included in Study 018 had at baseline predominantly lower scores of DRS, suggested that there was not an overexpression of patients with the phenotype of motor fluctuations predominantly of off-time. A post-hoc analysis of Study 016 and the SETLLE study confirmed the efficacy of safinamide in reducing the off-time compared with placebo [73]. Nonetheless, post-hoc analysis of study 018, stratifying patients on the presence/ absence of dyskinesia at baseline or changes in L-DOPA dose during the study, showed that safinamide $100 \mathrm{mg}$ improved the DRS score $(p=0.0488)$, but only among patients who did not change their L-DOPA dose [73].

In a more-recent double-blind, 6-month Phase III trial, NCT00627640 [72], safinamide (50-100 mg/day) was used as an add-on versus placebo in patients taking stable dosages of L-DOPA and concomitant PD medication. The study had the same inclusion criteria and outcomes as Study 016 and the SETTLE study, in which the researchers were instructed to in- crease the dose of safinamide up to $100 \mathrm{mg} /$ day, after day 14 . This study showed once again that safinamide was significantly effective in reducing off-time without troublesome dyskinesias. Yet, as in Study 018 and SETTLE, dyskinesia was still frequently reported as an $\mathrm{AE}$ [in 40 (14.6\%) versus 15 (5.5\%)] and as a severe $\mathrm{AE}$ [in $5(1.8 \%)$ versus $1(0.4 \%)$ ]. Thus, although the dose of $100 \mathrm{mg}$ /day provides the most-significant motor benefits, this might be at the expense of increased incidence of dyskinesias, at least in some patients. However, in line with the study design, the researchers were not allowed to reduce the dose of L-DOPA, raising the question of whether this would have reduced the incidence of dyskinesias.

In summary, there is clinical evidence to support that safinamide, mostly with an optimal dose of $100 \mathrm{mg}$ /day, can be effective in improving motor symptoms (reducing UPDRS III score) when used alone in early, untreated PD [65]. In intermediate-stage of PD $[71,73]$, and also favoring $100 \mathrm{mg} /$ day, there is strong evidence that safinamide, when used as an add-on therapy to a single DAA, leads to improvements in motor symptoms [68]. In advanced-stage PD with motor fluctuations, the addition of safinamide $50 \mathrm{mg} /$ day or $100 \mathrm{mg} /$ day to L-DOPA, as well as providing a symptomatic effect (reducing off-time and decreasing motor symptoms) $[69,70]$, is also effective in significantly increasing the total on-time with no or nontroublesome dyskinesia [58]. Furthermore, although supported in ad hoc subgroup analysis [70], safinamide might reduce the severity of dyskinesias in patients who already have moderate-severe dyskinesias over the long term (2 years of followup). There is no evidence that safinamide precludes the appearance of dyskinesias compared with L-DOPA. However, higher doses of safinamide ( $100 \mathrm{mg} /$ day) might aggravate the severity of dyskinesias. Moreover, robust evidence remains lacking for whether safinamide is able to delay the time to intervention [67], negating the need for L-DOPA.

\section{PD non-motor symptoms}

NMS, such as cognitive impairment and depression, are now recognized to be integral to $\mathrm{PD}$ during the prodromal phase and throughout the course of the disease, being a major contributor to the worsening of patient outcomes and quality of life [74,75]. Yet, there is still a paucity of effective therapies for NMS and the efforts of research in this field are lagging behind research on motor symptoms and complications. Currently, the only approved symptomatic drug for dementia in PD is rivastigmine, a cholinesterase inhibitor [76]. Although its symptomatic effect in dementia in PD and long-term safety have been established, there is no clear evidence for its use in mild cognitive impairment in PD (PD-MCI), or for its ability to halt disease progression [77]. Therefore, there are upcoming novel compounds expanding to the cognition field, with drugs targeting the glutamatergic, dopaminergic, noradrenergic, and serotonin systems [75]. In a trial using rasagiline, an improvement of patient-rated cognitive and depression outcomes was observed in patients with PD-MCI, highlighting the potential of this treatment [78]. However, subsequent trials of rasagiline, using neuropsychological assessment outcomes, failed to prove a meaningful benefit in cognition or depression [79]. The results of a multicenter Phase II (NCT01211587) doubleblind clinical trial using safinamide $100 \mathrm{mg} /$ day controlled with placebo in patients without dementia but with cognitive 
impairments, will further clarify the potential benefit of safinamide on cognition in PD.

In terms of PD-related depression, there are currently several clinical trials reinforcing its role in PD and, in contrast to other causes of depression, this could be the result of the larger range of dopaminergic deficits involved in PD. In fact, when dopamine agonists, such as rotigotine in the RECOVER study [80] and apomorphine [81], were applied, significant improvements in depression scores on the Beck Depression Inventory (BDI) and NMS scores were observed. Also with rasagiline, a post-hoc analysis revealed significantly less worsening of depression scores on the MDS-UPDRS experiences of daily living, compared with placebo after 36 weeks of follow-up in the ADAGIO study [78]. Notably, as recently reviewed by Finberg and Rabey [41], despite the strong antidepressant efficacy of irreversible MAO inhibitors, their clinical use has been limited because of their AEs in potentiating cardiovascular effects of dietary amines. Thus, the use of reversible MAO-B inhibitors, such as safinamide, have demonstrated a safer profile, thereby justifying further studies in PD with cognitive and/ or depression impairment.

\section{Concluding remarks}

Disease-modifying and/or neuroprotection effects are the Holy Grail for PD treatment, which, up to now, all antiparkinsonian drugs have failed to demonstrate. A disease-modifying effect reflects an intervention that modifies the natural clinical course of PD. Hopes were prematurely raised by the DATATOP trial [selegiline and selegeline + tocophenol (vitamin E) in patients with drug-naïve PD] [82], ADAGIO study (rasagiline in patients with drug-naïve PD) [83], SINDEPAR study [sinemet(levodopa/ carbidopa)-Deprenyl(selegeline)-Parlodel(bromocriptine)] [84], as well as by the SELEDO study (selegiline combined with L-DOPA in earlier stages of PD) [85], which all claimed to show that a proven delay in the need to start L-DOPA treatment resulted from a disease-modifying effect of the drugs involved. However, all these studies are plagued by the same issue, that these drugs (selegiline, rasagiline, and bromocriptine) have prodopaminergic effects. Thus, it is impossible to separate the confounding symptomatic benefits, which can have a carry-over motor effect over weeks to months, from any disease-modifying effects. Using neuroimaging outcomes with ${ }^{123}$ I-b-CIT SPECT in the CALM-PD study (pramipexole) [86], the authors suggested that this drug slowed the loss of DA-imaging markers relative to standard L-DOPA. Yet, these changes can be better interpreted in terms of drug-related DA regulation than a real structural neuroprotection, as shown in the PROUD-PD study [87]. Thus, we expect that, if one only takes into account its MAO-B inhibition effect, safinamide will have the same difficulties as all previous drugs in having a disease-modifying effect in PD. Nevertheless, these difficulties should not preclude further studies, especially in intermediate-stages of PD, before the development of motor fluctuations, to reinforce the hypothesis that safinamide, when used as an add-on therapy to LDOPA, is able to reduce its daily dosage, and eventually delay the incidence of MCs $[35,88]$. Also, in advanced stages of PD, with dyskinesias, future trials, or even ongoing clinical experiments, should determine whether the strategy of adding safinamide and lowering L-DOPA dosages will lead to a reduction in severe dyskinesias while simultaneously delivering the same motor benefits.
In terms of the MAO-B inhibition properties of safinamide, there are no studies comparing safinamide with rasagiline, selegiline, or entacapone; given the results from the LARGO study (rasagiline versus entacapone added to L-DOPA scheme) [89], we expect that the increased on-time without troublesome dyskinesias is in fact similar across the three drugs.

In contrast to disease-modifying effects, neuroprotection implies the protection of neurons from death or dysfunction resulting from the ongoing neuropathological processes; this is one of the unmet needs for PD, because current therapies do not address its degenerative progress [37]. Based on preclinical studies, safinamide has been shown to have neuroprotective and antiinflammatory effects in both in vitro and in vivo animals models of PD [37], although human data are still missing. The pleomorphic pharmacological properties of safinamide, especially its nondopaminergic properties (e.g., inhibition of voltage-dependent sodium channels and inhibition of stimulated glutamate) raise some hope for potential neuroprotective and disease-modifying effects on PD $[28,31]$.

In terms of NMS, further evidence is also needed to corroborate the potential benefit of safinamide on depression, as was suggested by the results of Study 18 [70], as well as on cognition, as suggested by the results of NCT01211587.

Magnetic resonance imaging (MRI) studies of the brain, in particular through functional MRI (fMRI), might be one of the best strategies to address several of these open questions. Functional MRI would allow one to explore the role of safinamide as a disease-modifying agent of motor and NMS of PD, especially its cognitive deficits and depressive symptoms, and to specify the neuronal circuits modulated by the drugs. We hypothesize that safinamide could enhance the effect of L-DOPA by acting through both dopaminergic and glutamatergic pathways. Therefore, patients with PD under concomitant safinamide treatment could show a different recruitment of limbic- and cognition-related brain circuits, which could be assessed through both resting-state (rs) and task-related fMRI in addition to the described effects of LDOPA in motor areas (Fig. 3). Notably, rs-fMRI has been shown to reveal functional reorganizations in PD through different approaches, both in patients with PD under dopamine-replacement therapies (DRT) and drug-naïve PD [90]. Particularly, patients with PD appear to present reduced corticostriatal functional connectivity and L-DOPA appears to partially normalize these functional connectivity imbalances. Thus, further connectomic analyses are needed to assess whether: (i) treatment with safinamide alone, or in combination with L-DOPA, has the ability to improve even further the normalization of these functional connectivity imbalances, to the same extent as L-DOPA (itself); and (ii) the improvements are associated with changes in network topology; and (iii) what other networks, besides the dopaminergic pathways, are involved.

Thus, satisfactory approaches to relieve the symptoms, or to slow down the progression, of PD by protecting DAn from premature death remain missing. Although promising results have been experimentally and clinically obtained with several drugs, the challenge is still to show a clinical proof of arrest or delay of DAn loss in PD [14]. In addition to DAn degeneration and DA depletion, multiple factors, including oxidative stress, mitochondrial dysfunction, excitotoxicity, and inflammation, have 
(a)
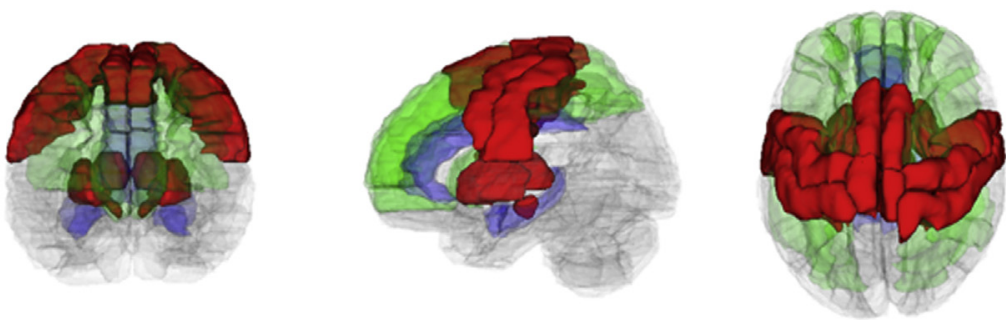

(b)
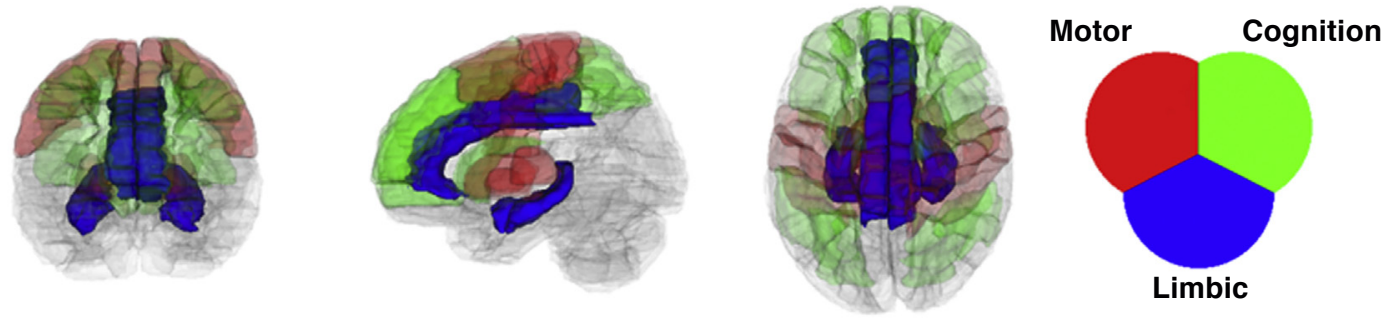

(c)
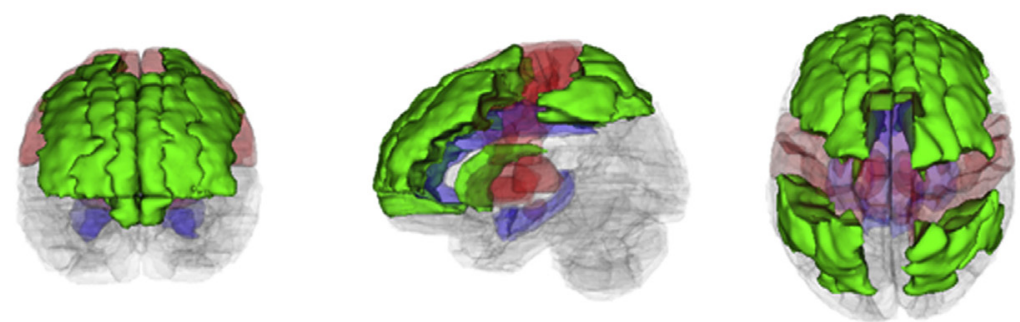

$\overline{\text { Drug Discovery Today }}$

\section{FIGURE 3}

Hypothesized brain areas modulated by safinamide. The dopaminergic action of safinamide appears to have an impact in (particularly) motor areas, similarly to the effects of levodopa (L-DOPA), thus reducing motor symptoms (a). Additionally, its nondopaminergic action might contribute to the reduction in non-motor symptoms, namely improving depressive symptoms by modulating the limbic system (b) or reducing cognitive impairment by modulating nondopaminergic circuits important for different cognitive functions (c).

also been described to be involved in the initiation and progression of PD [91]. This makes PD a multitargeted disease in which new strategies, with a multimodal action, could be of particular value $[31,44,92]$. Such a profile has been linked to safinamide, based on its high selective and reversible inhibition of MAO-B, leading to an increase in available DA (dopaminergic action of safinamide). In addition to dopaminergic transmission, safinamide has also been linked with nondopaminergic actions (e.g., neuroprotective and/or disease-modifying strategies $[14,28]$ ), blocking sodium channels and modulating the function of calcium channels, thereby inhibiting the release of glutamate [93]. Such properties show more potential compared with those of selegiline and rasagiline effects, although it is advised that all of the above-referred compounds should be taken daily. However, in the case of selegiline and rasagiline, there questionable results remain, because both drugs inhibit MAO-B in an irreversible manner [94]. In addition, it has been also claimed that a selegiline and rasagiline daily regimen might also cause inhibition of MAOA, although this awaits investigation in patients with PD. With safinamide, although more studies are needed to fully understood all its modes of action, several clinical trials have demonstrated its efficacy and tolerability, suggesting an optimal dosage raging from 50 to $100 \mathrm{mg} / \mathrm{day}$, correlated with an improvement in ontime and reduction in off-time. If a preventive strategy of motor complications with safinamide is possible, as defended by Thomas Müller [32,95], this will open the way for the wider use of safinamide for the prevention and treatment of motor complications. However, it will be necessary to investigate in more detail the impact of safinamide on L-DOPA pharmacokinetics with and without COMT inhibition in view of increasing L-DOPA levels during safinamide treatment. In addition to its effects on motor complications, safinamide might also have putative cognitionenhancing effects (although this needs to be proven) [14,95], making it an essential future PD drug, capable of modulating altered dopaminergic and glutamatergic neurotransmission in patients with PD. Therefore, future clinical trials and/or studies, especially with longer follow-up times, either in patients with de novo drug-naïve PD or with intermediate stages of PD (without MCs and/or cognitive impairment), could provide further enlightenment on these outstanding questions.

\section{Acknowledgments}

The authors acknowledge funding from the Portuguese Foundation for Science and Technology (IF development grant IF/ 00111/2013 to A.J.S.) and a postdoctoral fellowship to F.G.T.

(SFRH/BPD/118408/2016). This work was funded by FEDER funds, through the Competitiveness Factors Operational Programme (COMPETE), and by national funds, through the Foundation for 
Science and Technology (FCT), under the scope of the project POCI-01-0145-FEDER-007038.

This article has also been developed under the scope of the project NORTE-01-0145-FEDER-000023, supported by the Northern
Portugal Regional Operational Programme (NORTE 2020), under the Portugal 2020 Partnership Agreement, through the European Regional Development Fund (FEDER).

\section{References}

1 Michely, J. (2015) Dopaminergic modulation of motor network dynamics in Parkinson's disease. Brain 138, 664-678

2 Ascherio, A. and Schwarzschild, M.A. (2016) The epidemiology of Parkinson's disease: risk factors and prevention. Lancet Neurol. 15, 1257-1272

3 Hallett, M. (2003) Parkinson revisited: pathophysiology of motor signs. Adv. Neurol. 91, 19-28

4 Cano-de-la-Cuerda, R. et al. (2017) Axial rigidity is related to the risk of falls in patients with Parkinson's disease. NeuroRehabilitation 40, 569-577

5 Artigas, N.R. et al. (2016) Postural instability and falls are more frequent in Parkinson's disease patients with worse trunk mobility. Arq. Neuropsiquiatr. 74, 519 523

6 Matinolli, M. et al. (2011) Recurrent falls and mortality in Parkinson's disease: a prospective two-year follow-up study. Acta Neurol. Scand. 123, 193-200

7 Koychev, I. and Okai, D. (2017) Cognitive-behavioural therapy for non-motor symptoms of Parkinson's disease: a clinical review. Evid. Based Ment. Health 20, 15-20

8 Torbey, E. et al. (2015) Depression rating scales in Parkinson's disease: a critical review updating recent literature. J. Affect. Disord. 184, 216-224

9 Munhoz, R.P. et al. (2015) Non-motor signs in Parkinson's disease: a review. Arq. Neuropsiquiatr. 73, 454-462

10 Munhoz, R.P. et al. (2015) Non-motor signs in Parkinson's disease: a review. Arq. Neuropsiquiatr. 73, 454-462

11 Marques de Sousa, S. and Massano, J. (2013) Motor complications in Parkinson's disease: a comprehensive review of emergent management strategies. CNS Neurol. Disord. Drug Targets 12, 1017-1049

12 Diaz, N.L. and Waters, C.H. (2009) Current strategies in the treatment of Parkinson's disease and a personalized approach to management. Expert Rev. Neurother. 9, 1781 1789

13 Rajput, A. and Rajput, A.H. (2006) Parkinson's disease management strategies. Expert Rev. Neurother. 6, 91-99

14 Onofrj, M. et al. (2008) An expert opinion on safinamide in Parkinson's disease. Expert Opin. Investig. Drugs 17, 1115-1125

15 Lindholm, D. et al. (2016) Parkinson's disease: towards better preclinical models and personalized treatments. Cell. Mol. Life Sci. 73, 1383-1385

16 Dong, J. et al. (2016) Current pharmaceutical treatments and alternative therapies of Parkinson's disease. Curr. Neuropharmacol. 14, 339-355

17 Vijverman, A.C. and Fox, S.H. (2014) New treatments for the motor symptoms of Parkinson's disease. Expert Rev. Clin. Pharmacol. 7, 761-777

18 Krack, P. et al. (2017) Current applications and limitations of surgical treatments for movement disorders. Mov. Disord. 32, 36-52

19 Amalric, M. et al. (2013) Group III and subtype 4 metabotropic glutamate receptor agonists: discovery and pathophysiological applications in Parkinson's disease. Neuropharmacology 66, 53-64

20 Harikrishna Reddy, D. et al. (2014) Advances in drug development for Parkinson's disease: present status. Pharmacology 93, 260-271

21 Schneider, J.S. et al. (2015) GM1 ganglioside in Parkinson's disease: pilot study of effects on dopamine transporter binding. J. Neurol. Sci. 356, 118-123

22 Seet, R.C. et al. (2014) Does high-dose coenzyme Q10 improve oxidative damage and clinical outcomes in Parkinson's disease? Antioxid. Redox Signal. 21, 211-217

23 Tarazi, F.I. et al. (2014) Emerging therapies for Parkinson's disease: from bench to bedside. Pharmacol. Ther. 144, 123-133

24 Allen, P.J. and Feigin, A. (2014) Gene-based therapies in Parkinson's disease. Neurotherapeutics 11, 60-67

25 Garea-Rodriguez, E. et al. (2016) Comparative analysis of the effects of neurotrophic factors CDNF and GDNF in a nonhuman primate model of Parkinson's disease. PLoS One 11, e0149776

26 Di Santo, S. and Widmer, H.R. (2016) Paracrine factors for neurodegenerative disorders: special emphasis on Parkinson's disease. Neural Regen. Res. 11, 570-571

27 Espay, A.J. et al. (2017) Precision medicine for disease modification in Parkinson disease. Nat. Rev. Neurol. 13, 119-126

28 Sadeghian, M. et al. (2016) Neuroprotection by safinamide in the 6hydroxydopamine model of Parkinson's disease. Neuropathol. Appl. Neurobiol. 42, 423-435

29 Dezsi, L. and Vecsei, L. (2014) Safinamide for the treatment of Parkinson's disease. Expert Opin. Investig. Drugs 23, 729-742
30 Fabbri, M. et al. (2015) Clinical pharmacology review of safinamide for the treatment of Parkinson's disease. Neurodegener. Dis. Manag. 5, 481-496

31 Blair, H.A. and Dhillon, S. (2017) Safinamide: a review in Parkinson's disease. CNS Drugs 31, 169-176

32 Muller, T. (2016) Emerging approaches in Parkinson's disease - adjunctive role of safinamide. Ther. Clin. Risk Manag. 12, 1151-1160

33 Stocchi, F. and Torti, M. (2016) Adjuvant therapies for Parkinson's disease: critical evaluation of safinamide. Drug Des. Dev. Ther. 10, 609-618

34 Schnitker, J. and Müller, T. (2015) Meta-analysis of placebo-controlled clinical trials of safinamide and entacapone as add-on therapy to levodopa in the treatment of Parkinson's disease. Eur. Neurol. Rev. 10, 15-22

35 Muller, T. and Foley, P. (2017) Clinical pharmacokinetics and pharmacodynamics of safinamide. Clin. Pharmacokinet. 56, 251-261

36 Youdim, M.B. and Bakhle, Y.S. (2006) Monoamine oxidase: isoforms and inhibitors in Parkinson's disease and depressive illness. Br. J. Pharmacol. 147 (Suppl. 1), S287S296

37 Caccia, C. et al. (2006) Safinamide: from molecular targets to a new anti-Parkinson drug. Neurology 67 (Suppl. 2), S18-S23

38 Muller, T. (2017) Pharmacokinetic drug evaluation of safinamide mesylate for the treatment of mid-to-late stage Parkinson's disease. Expert Opin. Drug Metab. Toxicol. 13, 693-699

39 Robakis, D. and Fahn, S. (2015) Defining the role of the monoamine oxidase-B inhibitors for Parkinson's disease. CNS Drugs 29, 433-441

40 Binda, C. et al. (2007) Structures of human monoamine oxidase B complexes with selective noncovalent inhibitors: safinamide and coumarin analogs. J. Med. Chem. $50,5848-5852$

41 Finberg, J.P. and Rabey, J.M. (2016) Inhibitors of MAO-A and MAO-B in psychiatry and neurology. Front. Pharmacol. 7, 340

42 Marquet, A. et al. (2012) The effect of safinamide, a novel drug for Parkinson's disease, on pressor response to oral tyramine: a randomized, double-blind, clinical trial. Clin. Pharmacol. Ther. 92, 450-457

43 Cattaneo, C. et al. (2015) Long-term effects of safinamide on dyskinesia in mid- to late-stage Parkinson's disease: a post-hoc analysis. J. Parkinsons Dis. 5, 475-481

44 Cruz, M.P. (2017) Xadago (safinamide): a monoamine oxidase B inhibitor for the adjunct treatment of motor symptoms in Parkinson's disease. P. T. 42, 622-637

45 Cattaneo, C. et al. (2003) Pressor response to intravenous tyramine in healthy subjects after safinamide, a novel neuroprotectant with selective, reversible monoamine oxidase B inhibition. Clin. Neuropharmacol. 26, 213-217

46 Choy, M. (2017) Pharmaceutical approval update. P. T. 42, 366-371

47 Sampaio, C. and Ferreira, J.J. (2010) Parkinson disease: ADAGIO trial hints that rasagiline slows disease progression. Nat. Rev. Neurol. 6, 126-128

48 Palhagen, S. et al. (2006) Selegiline slows the progression of the symptoms of Parkinson disease. Neurology 66, 1200-1206

49 Olanow, C.W. et al. (2008) Why have we failed to achieve neuroprotection in Parkinson's disease? Ann. Neurol. 64 (Suppl. 2), S101-S110

50 Hart, R.G. et al. (2009) Neuroprotection trials in Parkinson's disease: systematic review. Mov. Disord. 24, 647-654

51 Rascol, O. (2009) 'Disease-modification' trials in Parkinson disease: target populations, endpoints and study design. Neurology 72 (7 Suppl), S51-58

52 Olanow, C.W. et al. (2008) A randomized, double-blind, placebo-controlled, delayed start study to assess rasagiline as a disease modifying therapy in Parkinson's disease (the ADAGIO study): rationale, design, and baseline characteristics. Mov. Disord. 23, 2194-2201

53 Parkinson Study Group (1989) DATATOP: a multicenter controlled clinical trial in early Parkinson's disease. Arch. Neurol. 46, 1052-1060

54 Fernandez, H.H. and Chen, J.J. (2007) Monamine oxidase inhibitors: current and emerging agents for Parkinson disease. Clin. Neuropharmacol. 30, 150-168

55 Group, P.S. (2005) A randomized placebo-controlled trial of rasagiline in levodopatreated patients with Parkinson disease and motor fluctuations: the PRESTO study Arch. Neurol. 62, 241-248

56 Peretz, C. et al. (2016) Comparison of selegiline and rasagiline therapies in parkinson disease: a real-life study. Clin. Neuropharmacol. 39, 227-231

57 Ahlskog, J.E. and Uitti, R.J. (2010) Rasagiline, Parkinson neuroprotection, and delayed-start trials: still no satisfaction? Neurology 74, 1143-1148 
58 Schapira, A.H. et al. (2017) Assessment of safety and efficacy of safinamide as a levodopa adjunct in patients with Parkinson disease and motor fluctuations: a randomized clinical trial. JAMA Neurol. 74, 216-224

59 Podurgiel, S. et al. (2013) Tremorolytic effects of safinamide in animal models of drug-induced parkinsonian tremor. Pharmacol. Biochem. Behav. 105, 105-111

60 Gregoire, L. et al. (2013) Safinamide reduces dyskinesias and prolongs L-DOPA antiparkinsonian effect in parkinsonian monkeys. Parkinsonism Relat. Disord. 19, 508-514

61 Morsali, D. et al. (2013) Safinamide and flecainide protect axons and reduce microglial activation in models of multiple sclerosis. Brain 136, 1067-1082

62 Tang, Y. and Le, W. (2016) Differential roles of M1 and M2 microglia in neurodegenerative diseases. Mol. Neurobiol. 53, 1181-1194

63 LeWitt, P.A. (2015) Levodopa therapy for Parkinson's disease: pharmacokinetics and pharmacodynamics. Mov. Disord. 30, 64-72

64 Ahlskog, J.E. and Muenter, M.D. (2001) Frequency of levodopa-related dyskinesias and motor fluctuations as estimated from the cumulative literature. Mov. Disord. 16, $448-458$

65 Stocchi, F. et al. (2004) Improvement of motor function in early Parkinson disease by safinamide. Neurology $63,746-748$

66 Stocchi, F. et al. (2012) A randomized, double-blind, placebo-controlled trial of safinamide as add-on therapy in early Parkinson's disease patients. Mov. Disord. 27 106-112

67 Schapira, A.H. et al. (2013) Long-term efficacy and safety of safinamide as add-on therapy in early Parkinson's disease. Eur. J. Neurol. 20, 271-280

68 Barone, P. et al. (2013) Safinamide as an add-on therapy to a stable dose of a single dopamine agonist: results from a randomized, placebo-controlled, 24-week multicenter trial in early idiopathic Parkinson disease (PD) patients (MOTION study) (P01.061). Neurology 80 (Suppl), P01.061

69 Borgohain, R. et al. (2014) Randomized trial of safinamide add-on to levodopa in Parkinson's disease with motor fluctuations. Mov. Disord. 29, 229-237

70 Borgohain, R. et al. (2014) Two-year, randomized, controlled study of safinamide as add-on to levodopa in mid to late Parkinson's disease. Mov. Disord. 29, 1273-1280

71 Schapira, A. et al. (2013) Safinamide significantly improves responder rates in fluctuating Parkinson's disease (PD) patients as add-on to levodopa (SETTLE). Mov. Disord. 28, S152

72 Schapira, A.H. et al. (2016) Assessment of safety and efficacy of safinamide as a levodopa adjunct in patients with Parkinson disease and motor fluctuations: a randomized clinical trial. JAMA Neurol. 74, 216-224

73 Cattaneo, C. et al. (2016) Safinamide as add-on therapy to levodopa in mid- to latestage Parkinson's disease fluctuating patients: post hoc analyses of Studies 016 and SETTLE. J. Parkinsons Dis. 6, 165-173

74 Hely, M.A. et al. (2008) The Sydney multicenter study of Parkinson's disease: the inevitability of dementia at 20 years. Mov. Disord. 23, 837-844

75 Schrag, A. et al. (2015) New clinical trials for nonmotor manifestations of Parkinson's disease. Mov. Disord. 30, 1490-1504

76 Francis, P.T. and Perry, E.K. (2007) Cholinergic and other neurotransmitter mechanisms in Parkinson's disease, Parkinson's disease dementia, and dementia with Lewy bodies. Mov. Disord. 22 (Suppl. 17), S351-S357
77 Emre, M. et al. (2014) Long-term safety of rivastigmine in parkinson disease dementia: an open-label, randomized study. Clin. Neuropharmacol. 37, 9-16

78 Smith, K.M. et al. (2015) Combined rasagiline and antidepressant use in Parkinson disease in the ADAGIO study: effects on nonmotor symptoms and tolerability. JAMA Neurol. 72, 88-95

79 Barone, P. et al. (2015) A randomized clinical trial to evaluate the effects of rasagiline on depressive symptoms in non-demented Parkinson's disease patients. Eur. J. Neurol. 22, 1184-1191

80 Ray Chaudhuri, K. et al. (2013) Rotigotine and specific non-motor symptoms of Parkinson's disease: post hoc analysis of RECOVER. Parkinsonism Relat. Disord. 19, 660-665

81 Martinez-Martin, P. et al. (2015) EuroInf: a multicenter comparative observational study of apomorphine and levodopa infusion in Parkinson's disease. Mov. Disord. 30, 510-516

82 Parkinson Study Group (1989) Effect of deprenyl on the progression of disability in early Parkinson's disease. N. Engl. J. Med. 321, 1364-1371

83 Olanow, C.W. et al. (2009) A double-blind, delayed-start trial of rasagiline in Parkinson's disease. N. Engl. J. Med. 361, 1268-1278

84 Stocchi, F. and Olanow, C.W. (2003) Neuroprotection in Parkinson's disease: clinical trials. Ann. Neurol. 53 (Suppl. 3), S87-S97 discussion S97-S89

85 Przuntek, H. et al. (1999) SELEDO: a 5-year long-term trial on the effect of selegiline in early Parkinsonian patients treated with levodopa. Eur. J. Neurol. 6, 141-150

86 Parkinson Study Group CALM Cohort Investigators (2009) Long-term effect of initiating pramipexole vs levodopa in early Parkinson disease. Arch. Neurol. 66, 563570

87 Schapira, A.H. et al. (2013) Pramipexole in patients with early Parkinson's disease (PROUD): a randomised delayed-start trial. Lancet Neurol. 12, 747-755

88 Cilia, R. et al. (2014) The modern pre-levodopa era of Parkinson's disease: insights into motor complications from sub-Saharan Africa. Brain 137, 2731-2742

89 Rascol, O. et al. (2005) Rasagiline as an adjunct to levodopa in patients with Parkinson's disease and motor fluctuations (LARGO, Lasting effect in Adjunct therapy with Rasagiline Given Once daily, study): a randomised, double-blind, parallel-group trial. Lancet 365, 9454-9947

90 Tahmasian, M. et al. (2015) A systematic review on the applications of resting-state fMRI in Parkinson's disease: does dopamine replacement therapy play a role? Cortex 73, 80-105

91 Yacoubian, T.A. and Standaert, D.G. (2009) Targets for neuroprotection in Parkinson's disease. Biochim. Biophys. Acta 1792, 676-687

92 Loprete, L. et al. (2016) Population pharmacokinetic and pharmacodynamic analyses of safinamide in subjects with Parkinson's disease. Pharmacol. Res. Perspect. 4, e00251

93 Morari, M. et al. (2017) Safinamide differentially modulates in vivo glutamate and GABA release in the rat hippocampus and basal ganglia. J. Pharmacol. Exp. Ther. XX, YYY-ZZZ

94 Dezsi, L. and Vecsei, L. (2017) Monoamine oxidase B inhibitors in Parkinson's disease. CNS Neurol. Disord. Drug Targets 16, 425-439

95 Muller, T. (2013) Current status of safinamide for the drug portfolio of Parkinson's disease therapy. Expert Rev. Neurother. 13, 969-977 